\title{
Representative elementary area for multifractal analysis of soil porosity using entropy dimension
}

\author{
F. San José Martínez ${ }^{1}$, F. J. Caniego ${ }^{1}$, C. García-Gutiérrez ${ }^{1}$, and R. Espejo ${ }^{2}$ \\ ${ }^{1}$ Dept. of Applied Mathematics to Agriculture Engineering, E.T.S.I Agrónomos, Technical University of Madrid (UPM), \\ 28040 Madrid, Spain \\ ${ }^{2}$ Dept. of Pedology, E.T.S.I Agrónomos, Technical University of Madrid (UPM), 28040 Madrid, Spain
}

Received: 2 February 2007 - Revised: 3 August 2007 - Accepted: 3 August 2007 - Published: 14 August 2007

\begin{abstract}
The notion of representative elementary area (REA) developed to address heterogeneity and scale problems in quantitative soil pedology comes from the notion of representative elementary volume of fluid dynamics in porous media. The REA allows the identification of the minimum area of a soil block section that is required to represent the pedofeature of interest based on its distribution in soil space. In this paper eight samples were imaged with two different techniques: the confocal microscope and the conventional film camera. These techniques provided information about pore sizes between $3.62 \mu \mathrm{m}$ and $161.98 \mu \mathrm{m}$, and between $39.72 \mu \mathrm{m}$ and $1776.34 \mu \mathrm{m}$, respectively. Sixteen of the resulting digital images were then analyzed to investigate the representative elementary area of the multifractal patterns of the spatial distribution of voids related to the micro and macroporosity by means of the entropy dimension. Our results permit the location of the REA region over the domain of the microstructures rendered by the analysis of the microscope images. They also suggest that this region seemingly spans scales of the macrostructures as revealed by the analysis of the camera pictures.
\end{abstract}

\section{Introduction}

Soil heterogeneity and complexity at several scales have been extensibility reported during recent decades. New mathematical tools based on fractals and multifractals have been put forward to capture these complex and highly irregular behaviors (see for instance Martin et al., 2005 and Martin et al., 2006, and the references therein). Soil porosity has been recognized as one of the pedofeatures that follows extremely irregular patterns which can be assess through multifractal analysis. Muller and McCauley (1992) were the first to characterize the multifractal behavior of the spatial arrangements

Correspondence to: F. San José Martínez

(fernando.sanjose@upm.es) of pore space in sedimentary rocks. Caniego et al. (2001) observed the same pattern for the soil pore size distribution.

Soil heterogeneity has been acknowledged as one of the main factors that hamper the ability of models based on Fick's law to describe transport phenomena in soil (Berkowitz et al., 2006). It has been suggested that water and solute migration through soil and aquifers exhibits features of anomalous or non-Fickian diffusion (Metzler and Klafter, 2004). In order to capture this behavior, new models based on continuous time random walks (Berkowitz et al., 2006) and fractional derivatives (Benson et al., 2000; Pachepsky et al., 2000) have been proposed. The aim of this work was to explore the notion of representative elementary area (REA) in this new context.

The notion of "representative elementary volume" (REV) introduced by Bear (1972) was the first step in passing from a microscopic level to a macroscopic one in order to develop a description of transport phenomena in multiphase porous media. It was defined as the minimum volume of a soil sample required from which a given soil parameter measurement becomes independent of the size of the sample. This volume is the representative elementary volume (REV). By analogy to the REV a "representative elementary area" (REA) may be also defined in order to deal with notions like fluxes or stresses that are associated with area in the context of transport phenomena in porous media. Bear and Bachmat (1984) concluded that for isotropic porous media the REV is well represented by the REA.

The REA has been applied to the study of heterogeneity and scale problems in quantitative soil micromorphology and pedology (Buchter et al., 1994; VandenBygaart and Protz, 1999; Bartoli et al., 2005). Here the goal is to find the minimum area on a soil block section that is required to represent the pedofeature of interest, based on its distribution in soil space (VandenBygaart and Protz, 1999). Hydrologists have also introduced this notion in the context of hydrologic modeling at the watershed scale (Woods et al., 1988; Blöschl

Published by Copernicus Publications on behalf of the European Geosciences Union and the American Geophysical Union. 
et al., 1995; Woods et al., 1995). Lin (2003) has showed how the REA and the REV appear to be key concepts in facilitating multiscale bridging when connecting pedology, soil physics and hydrology to integrate the pedon and landscape notions and to link phenomena at different scales: pores and aggregates, pedons and catenas and, watershed and regional scales.

Several works have explored the complex nature of soil porosity with multifractal analysis and they have established the suitability of this mathematical tool to characterize this highly irregular feature of soil with binarized images of sections of soil blocks (Muller and McCauley, 1996; Caniego et al., 2001; Posadas et al., 2003; Tarquis et al., 2003; GarcíaGutierrez and San José, 2004). Different techniques have been developed to acquire digitalized 2-D images. Scanning electron micrographs have been used by Muller and McCauley (1992). Tarquis et al. (2003) and Posadas et al. (2003) follow the procedure established by VandenBygaart and Protz (1999). Caniego et al. (2001) and GarcíaGutierrez and San José (2004) used an ultraviolet photographic technique that in the last cited work was refined with a confocal microscope. This list does not claim to be an exhaustive one.

The goal of this work was to identify a REA that would assess soil pore complex structure through the multifractal analysis of the voids of images of soil block sections using the entropy diemension. It has been suggested that the entropy dimension, one of the multifractal parameters, provides useful information when characterizing the irregular behavior of complex pedofeatures as the particle size or pore size distributions (Martin and Taguas, 1998; Caniego et al., 2001). It is also a physically meaningful parameter for its implications in measure, chaos and information theory (Martín and Taguas, 1998; Caniego et al., 2003). The entropy dimension has a twofold interpretation. From the point of view of information theory, it provides a way of quantifying the heterogeneity present in the distribution by the scaling pattern of the growth of the Shannon entropy as the scales shrink. From the point of view of the mathematical measure theory, it alludes to the geometrical size of pores where the distribution concentrates, in contrast with the box-counting dimension that gauges the geometric size of the spatial distribution of voids without considering the possible different concentrations (Caniego et al., 2003).

Two consecutive overlapping ranges of pore sizes were explored to identify the REA for soil block sections through the entropy dimension of the spatial arrangements of voids. We employed two different techniques of imaging sections of soil blocks hardened with fluorescent resins. One uses a confocal microscope and the other a digital camera. The confocal microscope provided information about pores which equivalent diameter (i.e. the diameter of a circle with the same area) lay between $3.62 \mu \mathrm{m}$ and $161.98 \mu \mathrm{m}$ while the camera rendered an interval which bounds were $39.72 \mu \mathrm{m}$ and $1776.34 \mu \mathrm{m}$.
This paper is organized as follows. The following section introduces the elements of the multifractal theory needed for the identification of a REA. Section 3 is devoted to the notion of REA. In Sect. 4 we overview the procedure for the preparation of samples, the image acquisition and binarization techniques, the estimation of the entropy dimension and the REA identification. We discus the results in Sect. 5 and present our conclusions in Sect. 6.

\section{Multifractal parameters and entropy dimension}

The multifractal analysis of a probability distribution on a rectangular region of the plane requires a set of different grids with rectangular cells. A common choice for the grids is to consider dyadic scaling down (Evertsz and Mandelbrot, 1992; Kravchenko et al., 1999): i.e., successive partitions of characteristic linear sizes $\varepsilon=2^{-k} L$, being $k=0,1,2, \ldots$ and $L$ the linear size of the region. At each size scale $\varepsilon$, a number $N(\varepsilon)=2^{2 k}$ of cells are considered and their respective measures $\mu_{i}(\varepsilon)$ are found from data. The number $\alpha_{i}(\varepsilon)=\log \mu_{i}(\varepsilon) / \log \varepsilon$ is the singularity or Hölder exponent of the $i$-th cell of size $\varepsilon$, i.e. the coarse singularity exponents. This exponent may be interpreted as a crowding index or a degree of concentration of $\mu$ : the greater this value the smaller the concentration of the measure and vice versa. It is, in fact, the logarithmic density of the $i$-th cell of the partition of characteristic size $\varepsilon$. Typically, coarse singularity exponents of multifractal distributions show a great variability, in such a way that in the limit $(\varepsilon \rightarrow 0)$ becomes a continuum filling up a whole interval $\left[\alpha_{\min }, \alpha_{\max }\right]$. In order to characterize the different scalings of the measure, the set $I_{\alpha}$ of points with singularity exponent equal to $\alpha$ is considered and its Hausdorff dimension $\operatorname{dim}_{H} I_{\alpha}$ computed. The function $f(\alpha)=\operatorname{dim}_{H} I_{\alpha}$ is called the singularity spectrum of the distribution $\mu$ and quantifies in geometrical and statistical sense the singular behavior of the measure. It gives the "sizes" of the sets where singularity exponents are located and it is related to the probability distribution of these exponents (see Evertsz and Mandelbrot (1992) for details).

The entropy dimension of the distribution may be defined by the expression

$D_{I} \approx \frac{\sum_{i=1}^{n(\varepsilon)} \mu_{i}(\varepsilon) \log \mu_{i}(\varepsilon)}{\log \varepsilon}$.

Here, the symbol " $\approx$ " means scaling or asymptotic behavior as $\varepsilon \rightarrow 0$ (Evertsz and Mandelbrot, 1992). The numerator of the right hand side of Eq. (1) is simply the Shannon entropy with respect to the grid of linear size $\varepsilon$ where the sum is taken over the cell with nonzero measure. So, the number $D_{I}$ quantifies the degree of heterogeneity of the distribution measuring the way the Shannon entropy scales as the linear size of the grid shrinks. On the other hand, the right hand side of Eq. (1) may be also considered as the average of logarithmic densities or concentrations of the distribution 
Table 1. Soil classification and selected properties of the studied soil samples.

\begin{tabular}{|c|c|c|c|c|c|c|c|c|}
\hline Sample & Soil classification & Parent material & $\mathrm{pH}$ & $\begin{array}{c}\text { Electrical } \\
\text { conductivity }\end{array}$ & $\begin{array}{c}\text { Organic } \\
\text { matter }\end{array}$ & Sand & Silt & Clay \\
\hline & & & & $\mu \mathrm{S} / \mathrm{cm}$ & \multicolumn{4}{|c|}{$\%$} \\
\hline 1 & Lithic Xerorthent & Gneiss & 7.5 & 341 & 3.3 & 61.2 & 21.5 & 17.3 \\
\hline 2 & Typic Xerorthent & Granite & 5.7 & 41 & 1.3 & 46.3 & 41.2 & 12.5 \\
\hline 3 & Typic Xerorthent & $\begin{array}{l}\text { Coluvium of } \\
\text { slates and schists }\end{array}$ & 6.1 & 171 & 4.87 & 56 & 36.8 & 7.2 \\
\hline 4 & Typic Xerufluvent & Low river terrace & 6.1 & 111 & 3.2 & 36.6 & 42.2 & 21.2 \\
\hline 5 & Dystric Xerorthent & Gneiss & 5.5 & 142 & 4.27 & 63 & 28.7 & 8.3 \\
\hline 6 & Typic Xerorthent & Granite & 5.9 & 32 & 1.13 & 83.7 & 10.2 & 6.1 \\
\hline 7 & Dystric Xerorthent & Gneiss & 4.7 & 91 & 5.8 & 68.5 & 25.3 & 6.2 \\
\hline 8 & Typic Xerorthent & Slates & 7.1 & 117 & 3.15 & 42.2 & 45.5 & 12.3 \\
\hline
\end{tabular}

weighted by $\mu$. It suggests that $D_{I}$ may be viewed as the expected value of the different concentrations when the distribution itself is taking into account. Then the right hand side of the above expression defines a singularity exponent, say $\beta$. It turns out that $D_{I}=f(\beta)=\operatorname{dim}_{H} I_{\beta}$ (Beck and Schlögl, 1995). Therefore, it may be said that the entropy dimension gauges the geometrical size of the set where the "main part" of the distribution concentrates.

\section{Representative elementary area}

In order to identify a REA for the pedofeature of interest the following conditions should be considered (Bear and Bachmat, 1984; Wood et al., 1988).

1. Three different domains should be distinguished on the length scales corresponding to the size of the soil matrix where the considered pedofeature is evaluated, i.e. $l<<D<<L$, where $l$ is the length scale characteristic of significant variation of the considered pedofeature, $L$ is the length scale of the slow variation or of the gross inhomogeneities and $D$ is the length scale of the REA.

2. The average values obtained over the REA must be independent of its size or vary only smoothly with increasing size of REA to ensure that they are statistically representative.

3. The REA should be indifferent with respect to the field property and should remain invariant or at least smoothly varying with time and location.

4. The average values should constitute continuous and differentiable fields over the temporal and spatial domains.
This work primarily focuses on the existence of the REA in accordance with points 1 and 2 above. We will consider the entropy dimension as a physically meaningful parameter to characterize the irregular patterns of soil porosity by imaging sections of soil blocks. In this investigation, $l, D$ and $L$ will correspond to the areal extend of the rectangular frames cropped from the digital images of the soil blocks to evaluate the entropy dimension.

\section{Overview of digital image acquisition and identifica- tion of the REA}

4.1 Sampling procedure and preparation of soil block sections

Undisturbed soil samples were collected in standard Kubiena tins $(60 \mathrm{~mm} \times 100 \mathrm{~mm} \times 43 \mathrm{~mm})$ from horizon $\mathrm{A}$ in the north of the Comunidad de Madrid in central Spain. The soils were not farmed for at least five years and have a slope less than $5 \%$ in all cases. In order to investigate the identification of the REA in a variety of soils eight samples with different pedological characteristics were selected for this work. The characteristics of these soils are gathered in Table 1. In the laboratory the samples were dried in an oven at 40 degrees Celsius for three days. Then they were immersed in a mixture of resin, polyester and styrene with the ultraviolet compound UVITEX OB (Ciba Geigy) in order to visualize the pores when using UV light. Methyl ethyl ketone peroxide and cobalt octoate were used as catalyst and activator, respectively, for the polymerization reaction (Murphy, 1986). Each of the hardened blocks were cut into a $1 \mathrm{~cm} \mathrm{sec-}$ tion with a rock saw and prepared as polished sections. Cuts were parallel to the soil surface. The resulting section sizes vary between $4.5 \mathrm{~cm} \times 5 \mathrm{~cm}$ and $5 \mathrm{~cm} \times 8 \mathrm{~cm}$ with a thickness of $0.5 \mathrm{~cm}$. 
Table 2. Sizes (pixels) of the frames cropped from the digital images; $\mathrm{M}$ - microscope images (they have all the same size); $\mathrm{C} 1, \ldots, \mathrm{C} 8$ camera images.

\begin{tabular}{cccccccccc}
\hline Frame & $\mathrm{M}$ & $\mathrm{C} 1$ & $\mathrm{C} 2$ & $\mathrm{C} 3$ & $\mathrm{C} 4$ & $\mathrm{C} 5$ & $\mathrm{C} 6$ & $\mathrm{C} 7$ & $\mathrm{C} 8$ \\
\hline 1 & $467 \times 468$ & $177 \times 259$ & $205 \times 241$ & $173 \times 225$ & $225 \times 209$ & $349 \times 149$ & $167 \times 217$ & $143 \times 203$ & $187 \times 249$ \\
2 & $933 \times 934$ & $353 \times 515$ & $409 \times 479$ & $343 \times 443$ & $449 \times 415$ & $697 \times 293$ & $33 \times 433$ & $285 \times 405$ & $373 \times 497$ \\
3 & $1397 \times 139$ & $8529 \times 771$ & $611 \times 715$ & $515 \times 667$ & $671 \times 619$ & $1045 \times 447$ & $499 \times 647$ & $427 \times 607$ & $557 \times 741$ \\
4 & $1863 \times 1864$ & $705 \times 1027$ & $815 \times 955$ & $685 \times 885$ & $895 \times 821$ & $393 \times 595$ & $663 \times 861$ & $567 \times 805$ & $743 \times 987$ \\
5 & $2329 \times 2330$ & $881 \times 1285$ & $1019 \times 1193$ & $857 \times 1107$ & $1119 \times 1033$ & $1741 \times 745$ & $829 \times 1075$ & $709 \times 1007$ & $927 \times 1231$ \\
6 & $2793 \times 2794$ & $1057 \times 1541$ & $1221 \times 1429$ & $1027 \times 1327$ & $1341 \times 1237$ & $2087 \times 891$ & $995 \times 1291$ & $851 \times 1209$ & $1113 \times 1479$ \\
7 & $3259 \times 3260$ & $1233 \times 1797$ & $1425 \times 1669$ & $1199 \times 1549$ & $1565 \times 1443$ & $2435 \times 1041$ & $1161 \times 1507$ & $993 \times 1409$ & $1297 \times 1723$ \\
8 & $3725 \times 3726$ & $1409 \times 2053$ & $1629 \times 1907$ & $1369 \times 1769$ & $1789 \times 1651$ & $2783 \times 1189$ & $1325 \times 1719$ & $1133 \times 1609$ & $1483 \times 1971$ \\
9 & $4189 \times 4190$ & $1585 \times 2309$ & $1831 \times 2143$ & $1541 \times 1991$ & $2011 \times 1855$ & $3131 \times 1337$ & $1491 \times 1935$ & $1275 \times 1809$ & $1667 \times 2215$ \\
10 & $4655 \times 4656$ & $1761 \times 2565$ & $2035 \times 2381$ & $1711 \times 2211$ & $2235 \times 2061$ & $3479 \times 1487$ & $1657 \times 2149$ & $1417 \times 2011$ & $1853 \times 2461$ \\
11 & $5119 \times 5120$ & $1937 \times 2821$ & $2237 \times 2617$ & $1881 \times 2429$ & $2457 \times 2265$ & $3825 \times 1633$ & $1821 \times 2361$ & $1557 \times 2209$ & $2037 \times 2705$ \\
\hline
\end{tabular}
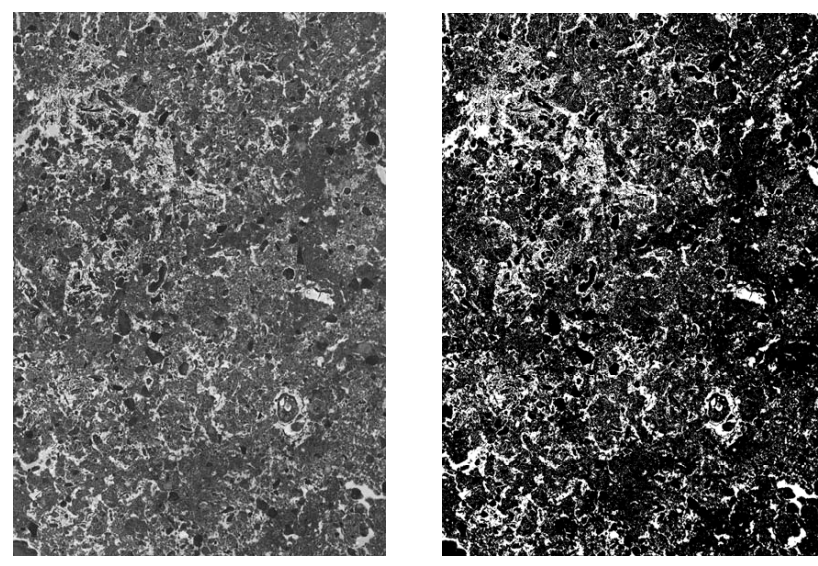

Fig. 1. Digital image of sample 1 obtained with camera, before (left) and after (right) binarization.

\subsection{Imaging of soil sections and binarization}

Two different instruments were used to image each soil section: a confocal microscope and a conventional film camera. In both cases, ultraviolet light was used when acquiring the pictures. The resulting digital pictures were 8-bit gray scale images.

Photographs were taken with a 100 ISO sensibility film with a frame size of $6 \mathrm{~cm} \times 6 \mathrm{~cm}$. The camera was $25 \mathrm{~cm}$ away from the sample. Digital images were obtained by scanning the film. The resulting resolution was $15.7 \mu \mathrm{m} /$ pixel. The resulting area of the rectangular images cropped from the pictures varied between $8.52 \mathrm{~cm}^{2}$ and $15.47 \mathrm{~cm}^{2}$.

The microscope used was a Leica TCS SP2 attached to a computer. The confocal technique permits the detection of light from the sample emitted by a single focal plane, excluding the light that comes from above or under that focal plane.
The pinhole from the microscope discriminates the light from the focal planes and the image obtained is not distorted by the adjacent planes. Ultraviolet light from two Argon lasers was adjusted to enhance the pore space domain taking into account the absorption spectrum of the Uvitex OB dye. A region of $3.75 \mathrm{~mm} \times 3.75 \mathrm{~mm}$ was chosen randomly from each soil section and it was imaged as a regular mosaic of $10 \times 10$ square tiles merged by the computer attached to the microscope. The resolution was $0.732 \mu \mathrm{m} /$ pixel.

As pointed out by Baveye et al. (1989) choosing of a threshold is a critical step in the binarization process, i.e. the operation performed on digital gray scale images to produce a binary image where each pixel is assigned one of two values, 0 for black and 1 for white. We used the method developed by Moran et al. (1989) based on the work of Serra (1982) on mathematical morphology. Figure 1 depicts the original digitalized picture (left) and the binarized one from the conventional film camera of one of the samples. Figure 2 corresponds to one of the images from the microscope. The left image is the original one and the right one is the binarized image. White colored regions correspond to pore space. These operations were performed with a macro developed for Inspector 4.1, imaging software of Matrox.

\subsection{Estimation of entropy dimension and REA identifica- tion}

From each of the sixteen binarized images (two for each of the eight soil sections) an experimental probability distribution $\mu$ is considered whose support is the whole rectangular domain of the picture. Binary partitions of each side of the rectangular domain were considered to get different grids with cells of linear size $\varepsilon=2^{-k} L$, being $k=0,1,2, \ldots$ and $L$ the length of one of the sides of the binarized image. Then, for any cell $I_{i}$ of size $\varepsilon, \mu\left(I_{i}\right)=\mu_{i}(\varepsilon)$ is the proportion of white colored pixel of the $I_{i}$ cell. A macro was developed for Inspector 4.1 to obtain the values of $\mu_{i}(\varepsilon)$ and estimate the corresponding entropy dimension. 
Table 3. Identification of the REA with the microscope images (M) with the size of the smallest frame of the REA region and the corresponding $j$-step in the series of cropped frames, and porosity of the images of the microscope (M) and the camera (C).

\begin{tabular}{llllll}
\hline Image & $j$-step & REA $\left(\mathrm{mm}^{2}\right)$ & Porosity $(\%)$ & Image & Porosity $(\%)$ \\
\hline M1 & 9 & 9.400 & 23.890 & C1 & 24.477 \\
M2 & 9 & 9.400 & 7.048 & C2 & 15.454 \\
M3 & 9 & 9.400 & 10.269 & C3 & 19.087 \\
M4 & 5 & 2.900 & 15.726 & C4 & 15.063 \\
M5 & 8 & 7.425 & 24.033 & C5 & 28.265 \\
M6 & 9 & 9.400 & 25.576 & C6 & 26.138 \\
M7 & 4 & 1.857 & 25.500 & C7 & 23.316 \\
M8 & 10 & 11.608 & 10.340 & C8 & 19.830 \\
\hline
\end{tabular}

The entropy dimension given by Eq. (1) was estimated by the linear fitting in a log-log plotting for decreasing values of $\varepsilon$. Points with coordinates $\log \varepsilon$ and $\sum_{i=1}^{n(\varepsilon)} \mu_{i}(\varepsilon) \log \mu_{i}(\varepsilon)$ were fitted to a line and coefficients of determination $R^{2}$ were recorded.

To establish the variation of the entropy dimension with scale, a sequence of eleven increasingly larger frames were cropped from each of the sixteen digital binarized images. The frame sequence was selected as follows. Ten points equally distant from each other were selected on the line connecting one corner with the center of the image. Each of these points was one of the corners of one frame which center was the center of the image. They were rectangular for the pictures of the camera and squared in the case of the microscope. Table 2 gathers the information about the sizes of these frames. Following the procedure established above the entropy dimension was estimated for each of the eleven frames on the sixteen images. They were estimated with four partitions for the smallest frames and seven for the largest ones of the microscope images. In the case of the pictures of the camera the smallest frames provided three or four partitions while the larges ones facilitated between five and seven. A series of values $D_{I}^{j}$, for $j=1,2, \ldots, 11$, were obtained for each image corresponding to entropy dimensions. The last value corresponds to the full image. We had considered the relative increment $R I_{j}$ in the entropy dimension of the $j$-step of each series, i.e. for $D_{I}^{j}$ the relative increment to the previous smaller frame area is $R I_{j}=\left|\left(D_{I}^{j}-D_{I}^{j-1}\right) / D_{I}^{j-1}\right| \times 100$. And we had compared them with the coefficient of variation $C V$ of the series, i.e. if $\mu$ and $\sigma$ are the mean and the standard deviation of the series it is $C V=(\sigma / \mu) \times 100$. For each image, the REA was identified by the triple $l, D$ and $L$ (see Sect. 3). Then, the region $D$ bounded by $l$ and $L$ corresponds to the range of scales where the relative increments of the values of the series $D_{I}^{j}$ of the entropy dimensions were less than $20 \%$ of the $C V$, i.e. $R I_{i} \leq 0.2 C V$.
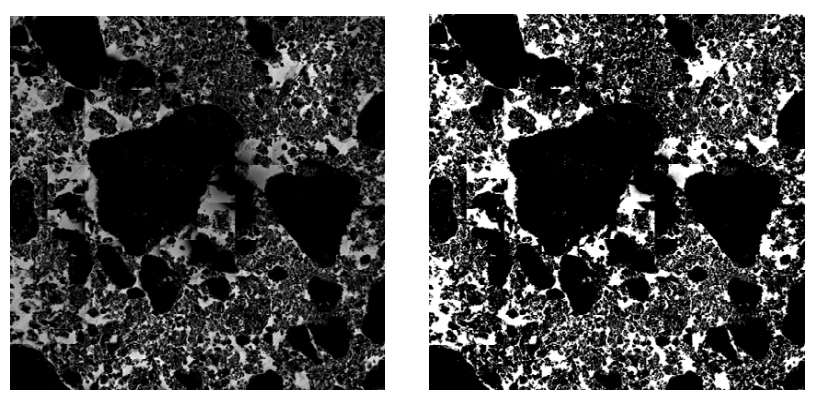

Fig. 2. Digital image of sample 1 obtained with microscope, before (left) and after (right) binarization.

\section{Results and discussion}

The coefficients of determination of the entropy dimension $R^{2}$ were greater than 0.99 (data not shown). Figsures 3 and 4 depict the series of the eleven estimated values of the entropy dimension against the area $\left(\mathrm{cm}^{2}\right)$ of the corresponding frame cropped from each image.

Two different regions in the microscope series can be distinguished (see Figs. 3 and 4), i.e. a range of scales with apparently significant variation followed by a plateau like region (see Figs. 3 and 4). In contrast, there is seemingly only one kind of plateau like range of scales when the camera images are considered. In fact (see Fig. 5), the coefficient of variation is always smaller for the series of entropy dimensions corresponding to the camera images. Moreover, while the relative increments of the macroporosity series (camera images) is always less than the $20 \%$ of coefficient of variation, this is only verified by the relative increments of the microporosity series (microscope images) from the terms with an open circle in Figs. 3 and 4. Table 3 gathers information about the lower bound of the REA region $D$ : the corresponding location in the series ( $j$-step), its entropy dimension and areal size (in all cases the upper bound $L$ were greater than the size of the image), and the image porosity as the percentage of white pixels. Therefore, it might suggest that in the microporosity domain there is a range of scales characteristic of rapid variation of its entropy dimensions followed by a region of scales with slowly varying quantities corresponding to the REA domain. Moreover, this domain also spans macroporosity scales. Taking one sample or soil block section, the graphic of the porosity entropy dimensions as a function of the areal frames for the two images (microscope image and camera image) seems to indicate that they compose a sequence of consecutive overlapping domains of different scales.

Figure 6 depicts the mean values of the entropy dimension on the REA region of the microporosity and the macroporosity of each sample. The means for macroporosity were significantly higher than the means of microporosity, except for the sample four. This fact might be a new indication of 

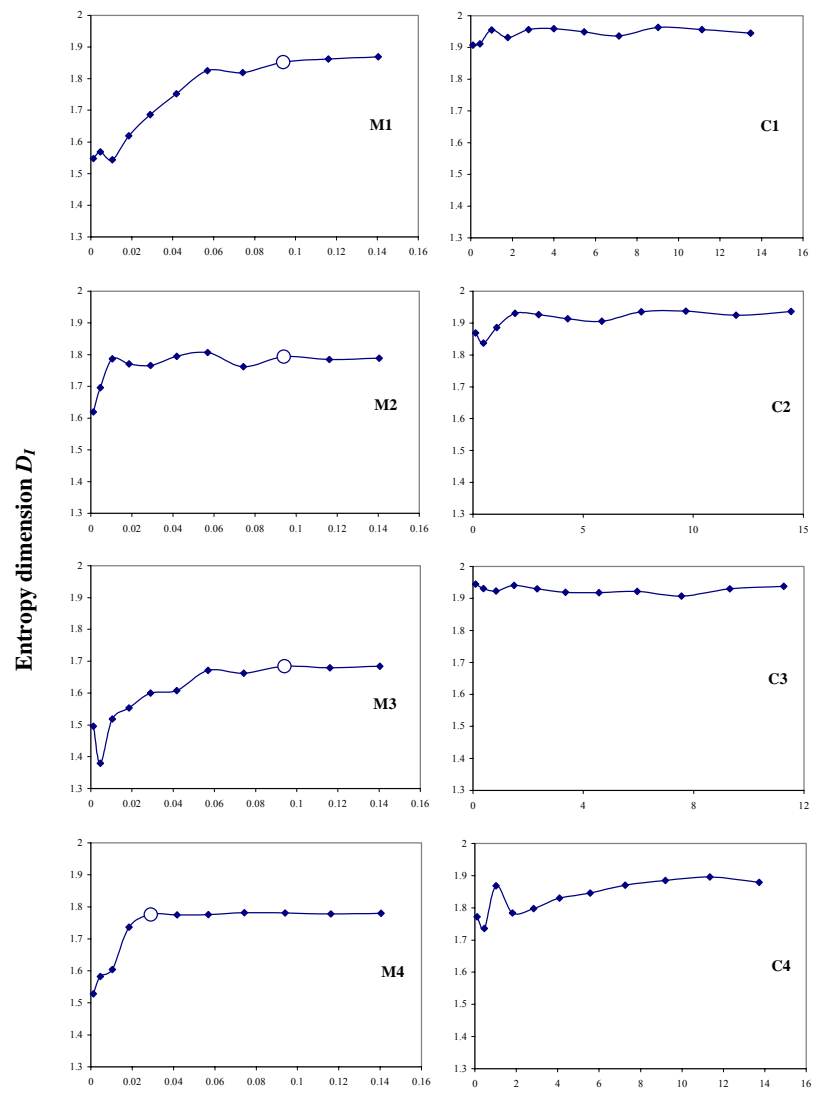

Area $\left(\mathrm{cm}^{2}\right)$

Fig. 3. Entropy dimensions $D_{I}$ for samples $1,2,3$ and 4 plotted against the area $\left(\mathrm{cm}^{2}\right)$ of the frames for the images of the microscope (M) and the camera (C). The left bound of the REA region is identify with an open circle.

the bimodal structure of soil porosity that has been reported when fractal scaling of soil porosity has been investigated (Anderson et al., 1996; Bartoli et al., 2005; Menéndez et al., 2005). As far as we know, such an effect has not been reported in the context of multifractal analysis of soil porosity. It is possible that this lack on previous analysis is due to the fact that, depending on the imaging technique, the microstructures and macrostructures that have been investigated with this mathematical tool have always been treated separately. Another explanation for the different values of the averages of entropy dimensions is that, due to the lower resolution of the of the camera images, the smallest scale ranges were not considered and, thus, finer structures of soil porosity were hidden. Thus, it could be assumed that these finer and complex patterns would render a lower entropy dimension than the one estimated with the camera images. In these images only the macrostructures of the porosity can be assessed. Contrast of this hypothesis would imply the imaging of the full soil section with a resolution similar to the resolution of the confocal microscope. The microscope images
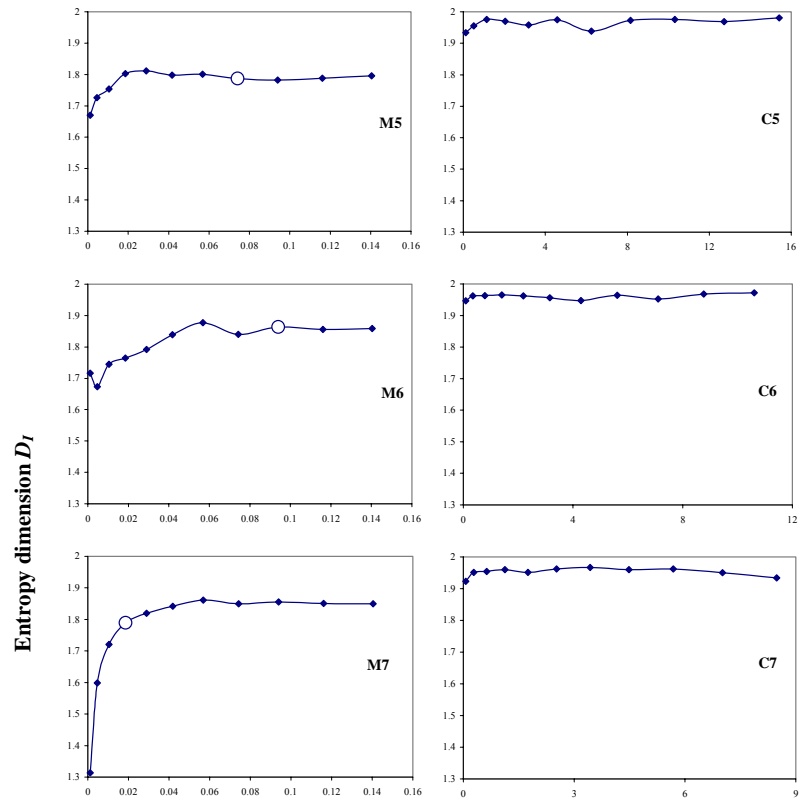

C6
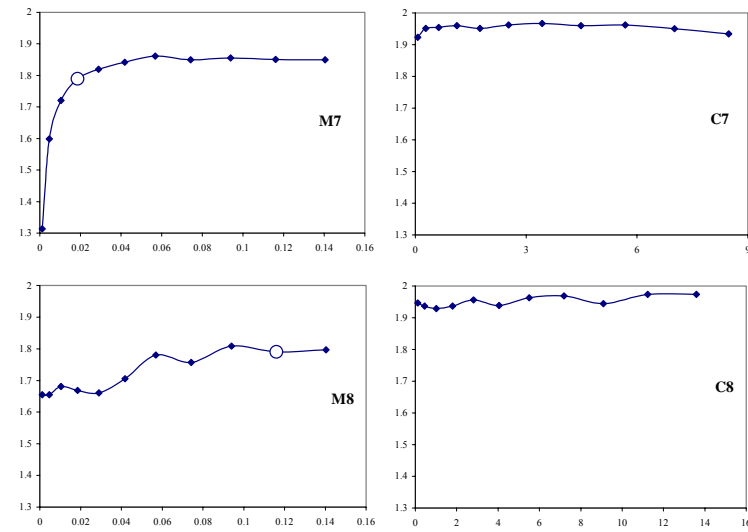

Area $\left(\mathrm{cm}^{2}\right)$

Fig. 4. Entropy dimensions $D_{I}$ for samples 5, 6,7 and 8 plotted against the area $\left(\mathrm{cm}^{2}\right)$ of the frames for the images of the microscope $(\mathrm{M})$ and the camera $(\mathrm{C})$. The left bound REA region is identify with an open circle.

are squares of $3.75 \mathrm{~mm}$ with a resolution of $0.734 \mu \mathrm{m} /$ pixel composed by merging of $10 \times 10$ frames. The total size that the computer needed to handle was about 400 megabytes and the time needed to acquire a single image was about $40 \mathrm{~min}$ plus the time for the location of the sample and the adjustment of the imaging software. To obtain an image with a linear size ten times larger $(3.75 \mathrm{~cm})$ with the same resolution these numbers should be multiplied by a factor of 100 . For the moment there is a clear limitation to the acquisition of images of this size at this higher resolution.

It might be also argued that the difference in the entropy dimension for the microscope and the camera images of the same block section could be an experimental artifact as a consequence of poor resin penetration or the limitation in the spatial resolution imposed by the image acquisition procedure. The camera image allows characterizing of the macrostructures. These images contain what Moran et al. (1989) call class III pores. They are neither large enough to appear sharp nor so tiny that they cannot be seen. Therefore, they appear somehow fuzzy in the image. The segmentation process they follow and we use is intended to 
separate them from the image background, i.e. the soil matrix in this case. As stated by Moran et al. (1989) and Giménez et al. (1997) class III pores are related to the image resolution or poor resin penetration but this does not result in a methodology deficiency when characterizing pores at meso/macro scale (Giménez et al., 1997). We have used a different technique to characterize pores at micro scale. The confocal microscope allows the acquisition of images that do not display class III pores. Then, even if there is no method that will result in resin-penetration of all soil pores, our results suggest that, at the resolution of the microscope, the pores with incomplete resin penetration are separated from the soil matrix (the image background).

Bird et al. (2006) has studied the constraints that the image porosity imposes on the values of the fractal and multifractal parameters. In this context the porosity of a digital binarized image is understood as the proportion of white pixel on the image; in this study white pixels correspond to pore space. In particular, when the porosity of the image is $f$, Bird et al. (2006) showed that for the grid size $\varepsilon$ the Shannon entropy for this partition is bounded by

$2 \ln \left(\frac{L}{\varepsilon}\right)+\ln f \leq-\sum_{i=1}^{n(\varepsilon)} \mu_{i}(\varepsilon) \log \mu_{i}(\varepsilon) \leq 2 \ln \left(\frac{L}{\varepsilon}\right)$

Thus, the value of the Shannon entropy of each partition size belongs to a band delimited by two lines with slope equal to two and vertical width equal to $-\ln f$. Let us examine expression (2). There are two extreme cases: when the porosity is 1 the bandwidth is zero and the Shannon entropy attains its maximum. In fact what we have here is the uniform distribution. In this situation there is only one way to spread the mass of the distribution, i.e. there is only one possible arrangement of voids within the image because the whole image is white. When the porosity decreases the bandwidth increases (it goes to infinity as the porosity goes to zero) with "speed" equal to $-1 / f$. This reflects the fact that when the porosity decreases there are more ways that the mass distribution of the pore space can be spread over the image, and thus the entropy dimension is one way to gauge these different types of spreading. In this investigation images have an intermediate or low porosity (see Table 3 ). Image C5 has the largest value with a porosity of 0.28 and M2 the lowest with a porosity of 0.07. Porosity was larger for the camera images, except for sample seven. Therefore the bandwidth that constrains the Shannon entropy for each partition will be smaller for the camera images with the exception mentioned above and it should be expected that the slope of the fit would be more likely to be closer to two for the camera images than for the images from the microscope. These comments suggest that the higher entropy dimension of the camera images could be ascribed to its higher image porosity. But, Figs. 7 and 8 seem to support the fact that there was not any pattern that relates entropy dimension and porosity in theses cases, neither for microporosity nor for macroporosity. Figure 8 suggests that the entropy dimension of the camera images with lower

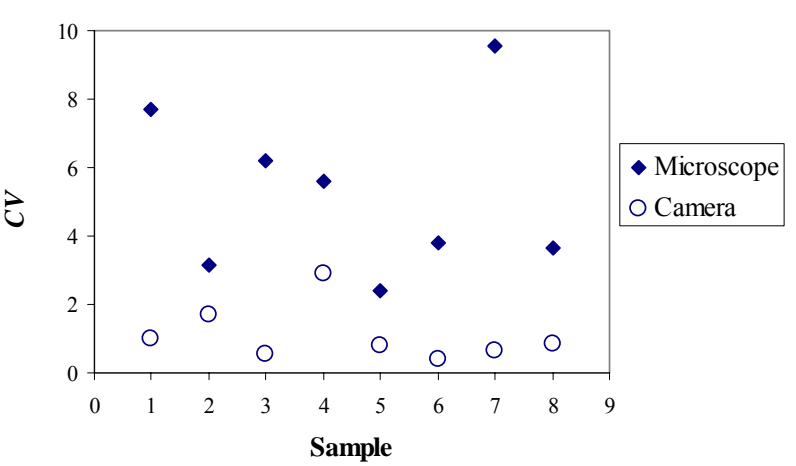

Fig. 5. $C V$ for the eleven frames of the images from the microscope and the camera.

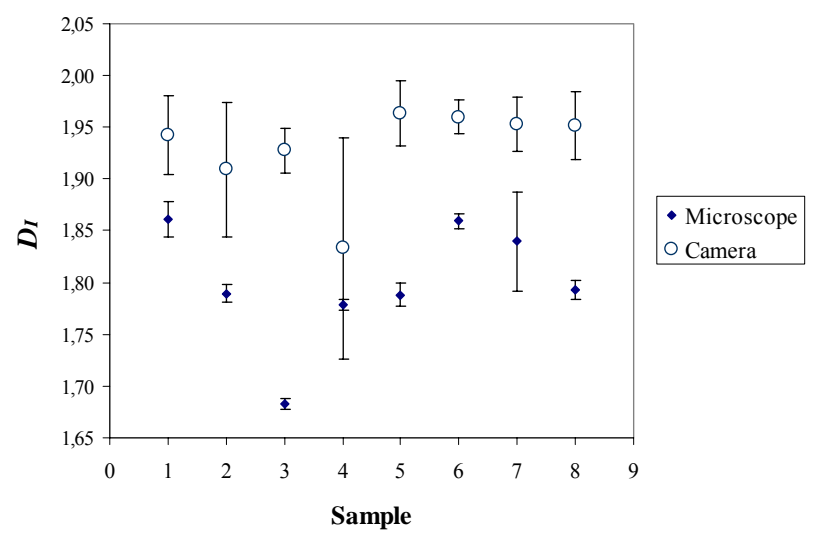

Fig. 6. Mean entropy dimension, $D_{I}$, on the REA region identified with the microscope images and with the camera images.

porosity were significantly different while they were more similar when the porosity was higher. On the contrary, there was not any clear trend in the case of the microstructures (see Fig. 7). As a consequence, there was no evidence that the results of Bird et al. (2006) could affect the conclusions of this investigation.

Following VandenBygaart and Protz (1999), our results would imply that the size of the microscope images used to represent the multifractal pattern of soil microporosity, as well as the size of the camera images used to assess macroporosity, is sufficiently satisfactory, when the hypothesis of bimodal behavior is assumed. If this hypothesis is not assumed, further investigations with powerful instruments are required to perform the multifractal analysis of the full scales of soil porosity to estimate its entropy dimension through image analysis of soil block sections.

\section{Conclusions}

Sections of eight soil blocks hardened with fluorescent resins were imaged with two different techniques. The confocal 


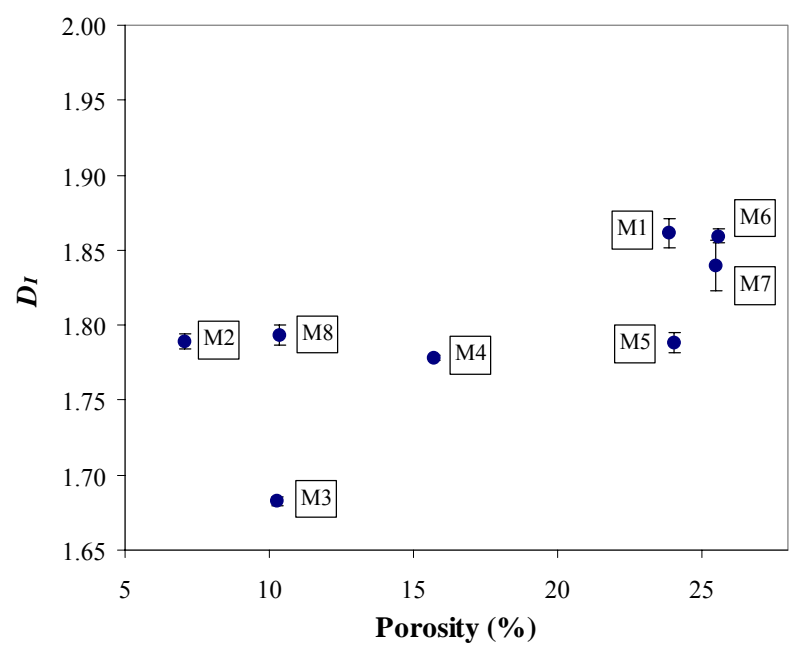

Fig. 7. Mean entropy dimension, $D_{I}$, with standard error bars of the REA region identified with the microscope images $(\mathrm{M})$ and porosity $(\%)$.

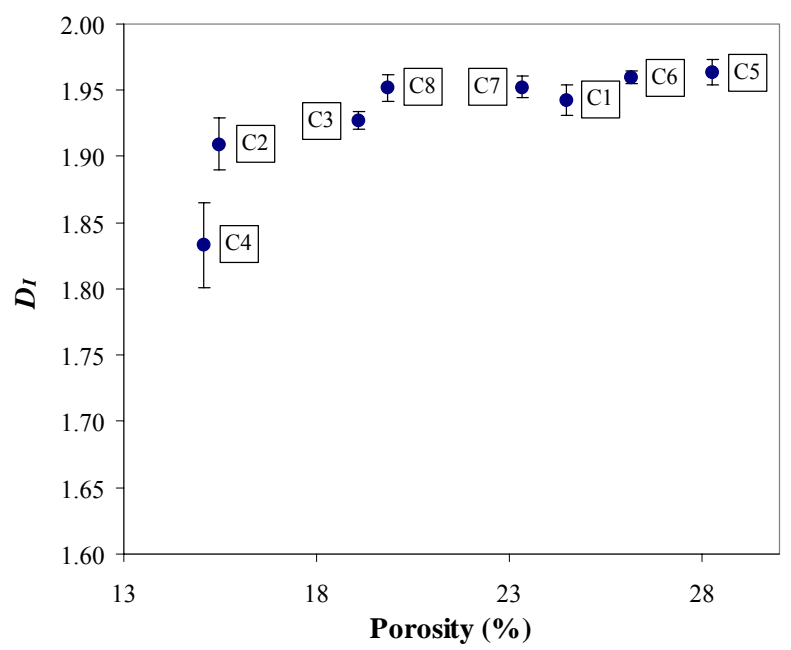

Fig. 8. Mean entropy dimension, $D_{I}$, with standard error bars of the camera images (C) and porosity $(\%)$.

microscope provided information about pores with an equivalent diameter between $3.62 \mu \mathrm{m}$ and $161.98 \mu \mathrm{m}$, whilst the camera rendered an interval of pore diameters whose boundaries were $39.72 \mu \mathrm{m}$ and $1776.34 \mu \mathrm{m}$. Sixteen digital images were analyzed to investigate the representative elementary area of the multifractal patterns of the spatial distribution of voids related to the microporosity and to the macroporosity.

The entropy dimension was selected as a physically meaningful parameter to assess the REA of the multifractal scaling of soil porosity. The REA was identified as the domain of scales of slow variation of the porosity entropy dimension.

Our results allow location of the REA region within the domain of the microstructures rendered by analysis of the images acquired with the confocal microscope. They also suggest that this region seemingly spans scales of the macrostructures explored by the analysis of the pictures of the camera. Then, the graphic of the porosity entropy dimension as function of areal frame for the two images seem to indicate that they compose a sequence of consecutive overlapping domains of different scales for each sample.

Two significantly different averaged values of the porosity entropy dimension were obtained for the REA domain. The entropy dimension of the macroporosity was higher. This difference might reflect the bimodal structure of soil porosity that has been reported when scaling analysis of soil porosity has been investigated. Such an effect does not seem to be reported in the context of multifractal analysis of soil porosity through image analysis of soil sections. This omission is probably due to the fact that, depending on the imaging technique, microstructures and macrostructures had previously been separately investigated with this mathematical tool. Another possible explanation of the differences between the average values of the estimated entropy dimensions over the REA domain could be related to the fact that the smallest scales were absent in the images from the camera. Thus, it could be assumed that these finer and complex patterns would render a lower entropy dimension than the one estimated with the camera images.

Bird et al. (2006) have considered how the image porosity imposes constraints on the values of the entropy dimension. The Shannon entropies used to estimate the entropy dimension must lie within a band delimited by two lines with slope equal to 2 and vertical width equal to the absolute value of the logarithm of the porosity. Thus, it should be expected that the slope of the fit to estimate the entropy dimension from the Shannon entropies would be more likely to be closer to 2 for images with high porosity. It suggests that the higher entropy dimension of the camera images could be ascribed to its higher porosity.

From the point of view of VandenBygaart and Protz (1999) the size of the images of the microscope permit the representation of the multifractal pattern of soil microporosity and the same is true for the size of the camera images with respect to soil macroporosity, when the hypothesis of the bimodal behavior is assumed. If this hypothesis is not assumed further investigations with powerful instruments are required to perform the multifractal analysis of the full scales of soil porosity to estimate its entropy dimension through image analysis of soil block sections.

Acknowledgements. This work was partially supported by Plan Nacional de Investigación Científica, Desarrollo e Investigación Tecnológica (I+D+I) under ref. AGL2004-04079 AGR. Spain and DGUI (Comunidad de Madrid) and UPM (project ref.: M0700204135). We would like to express our gratitude to M. A. Martín Martín for enlighten and stimulating discussions.

Edited by: A. Tarquis

Reviewed by: D. Gimenez and another anonymous referee 


\section{References}

Anderson, A. N., McBratney, A. B., and FitzPatrick, E. A.: Soil mass, surface, and spectral fractal dimensions estimated from thin section photographs, Soil Sci. Soc. Am. J., 60, 962-969. 1996.

Bartoli, F., Genevoise-Gomendy, V., Royer, J. J., Niquet, S., Vivier, H., and Grayson, R.: A multiscale study of silty soil structure, European J. Soil Sci., 56, 207-223, 2005.

Baveye, P., Boast, C. W., Ogawa, S., Parlange, J.-Y., and Stenhuis, T.: Influence of image resolution and thresholding on the apparent mass fractal characteristics of preferential flow patterns in field soils, Water Resour. Res., 34, 2783-2796, 1998.

Bear, J.: Dynamical of fluids in porous media. Dover Pub. Inc, New York, 1972.

Bear, J. and Bachmat, Y.: Transport in porous media. Basic equations, in: Fundamentals of transport phenomena in porous media, edited by: Bear, J. and Corapeioglu, M. Y., NATO ASI series, M. Nijhoff, Dordrecht, 3-61, 1984.

Beck, C. and Schlögl, F.: Thermodynamics of chaotic systems. An Introduction. Cambridge University Press, New York, 1995.

Benson, D. A., Wheatcraft, S. W., and Meerschaert, N. M.: Application of a fractional advection-dispersion equation, Water Resour. Res., 36(6), 1403-1412, 2000.

Berkowitz, B., Cortis, A., Dentz, M., and Scher, H.: Modeling non-fickian transport in geological formations as a continuous time random walk, Rev. Geophys., 44, RG2003, doi:10.1029/2005RG000178, 2006.

Bird, N., Díaz, M. C., Saa, A., and Tarquis, A. M.: Fractal and multifractal analysis of pore-scale images of soil, J. Hydrol., 322, 211-219, 2006.

Blöschl, G., Grayson, R. B., and Sivapalan, M.: On the representative elementary area REA. Concept and its utility for distributed rainfall-runoff modelling, in: Scale Issues in Hydrological Modelling, edited by: Kalma, J. D. and Sivapalan, M., Advances in Hydrological Processes. Wiley, Sussex, England, 71-88, 1995.

Buchter, B., Hinz, C., and Flühler, H.: Sample size for determination of coarse fragment content in stony soil, Geoderma, 63, 265-275, 1994.

Caniego, F. J., Martín, M. A., and San José, F.: Singularity features of pore- size soil distribution: singularity strength analysis and entropy spectrum, Fractals, 6, 305-316, 2001.

Caniego, F. J., Martín, M. A., and San José, F.: Rényi dimensions of soil pore size distribution, Geoderma, 112, 205-216, 2003.

Everstz, C. J. G. and Mandelbrot, B B.: Multifractal measures. In Peitgen, H., H. Jürgens, and D. Saupe. Chaos and Fractals, Springer, Berlin, 921-953, 1992.

García-Gutierrez, C. and San José Martínez, F.: Quality image acquisition and processing for multifractal characterization of soil porosity. International workshop on fractal mathematics applied to soil and related heterogeneous systems. El Barco de Avila, Spain, July 2-6, 2004.

Giménez, A., Allmaras, R. R., Nater, E. A., and Huggins, D. R.: Fractal dimension for volume and surface interaggregate pores scale effects, Geoderma, 77, 19-38, 1997.
Kravchenko, A. N., Boast, C. W., and Bullock, D. G.: Multifractal analysis of soil spatial variability, Agron. J., 91, 1033-1041, 1999.

Lin, H.: Hydropedology: Bridging Disciplines, Scales, and Data, Vadose Zone Journal 2, 1-11, 2003.

Martín, M. A. and Taguas, F. J.: Fractal modelling, characterization and simulation of particle-size distribution in soil, Proc. R. Soc. Lond. A, 454, 1457-1468, 1998.

Martin, M. A., Perfect, E., and Pachepsky, Y. (Eds.): Scaling fractals and diversity in soil and ecohydrology, Ecological Modelling, 182, 217-384, 2005.

Martin, M. A., Perfect, E., and Pachepsky, Y. (Eds.): Fractal geometry applied to soil and related hierarchical systems. Fractals, complexity and heterogeneity, Geoderma, 134, 237-456, 2006.

Menéndez, I., Caniego, J., Gallardo J. F., and Olechko, K.: Use of fractal scaling to discriminate between and macro- and mesopore size in forest soil. Ecological Modelling, 182, 323-335, 2005.

Metzler, R. and Klafter, J.: The restaurant at the end of the random walk: recent developments in the description of anomalous transport by fractional dynamics. J. Phys. A: Math. Gen., 37, R161R208, doi:10.1088/0305-4470/37/31/R01 J, 2004.

Moran, C. J., McBratney, A. B., and Koppi, A. J.: A rapid method for analysis of soil macropore structure. I. Specimen preparation and digital binary image production, Soil Sci. Soc. Am. J. 53, 921-928, 1989.

Muller, J. and McCauley, J. L.: Implication of fractal geometry for fluid flow properties of sedimentary rocks, Transport in Porous Media, 8, 133-147, 1992.

Murphy, C. P.: Thin section preparation of soils and sediments. AB Academic Pub., Berkhmasted, UK, 1986.

Pachepsky, Y. A., Benson, D. A., and Rawls, W.: Simulating scaledependent solute transport in soils with the fractional advectivedispersive equation, Soil Sci. Soc. Am. J., 64, 1234-1243, 2000.

Posadas, A. N. D., Giménez, D., Quiroz, R., and Protz, R.: Multifractal characterization of soil pore systems, Soil Sci. Soc. Am. J., 67, 1361-1369, 2003.

Serra, J.: Introduction to mathematical morphology. Academic Press, London, 1986.

Tarquis, A. M., Giménez, D., Saa, A., Díaz, M. C., and Gascó, J. M.: Scalings and multiscalings of soil pore systems determine by image analysis. In: Pachepsky, Y., D.E. Radcliffe and H. M. Selim (Editors). Scalings methods in soil physics. CRC Press, Boca Raton, Florida, USA, 19-33, 2003.

VandenBygaart, A. J. and Protz, R.: The representative elementary area (REA) in studies of quantitative soil micromorphology, Geoderma, 89, 333-346, 1999.

Wood, E. F., Sivapalan, M., Beven, K., and Band, L.: Effects of spatial variability and scale with implications to hydrologic modeling, J. Hydrol., 102, 29-47, 1988.

Woods, R. A., Sivapalan, M., and Duncan, M.: Investigating the representative elementary area concept: an approach based on field data, in: Scale Issues in Hydrological Modeling, edited by: Kalma, J. D. and Sivapalan, M., Advances in Hydrological Processes. Wiley, Sussex, England, 49-70, 199. 